\title{
Probing hidden sectors with a muon beam: Total and differential cross sections for vector boson production in muon bremsstrahlung
}

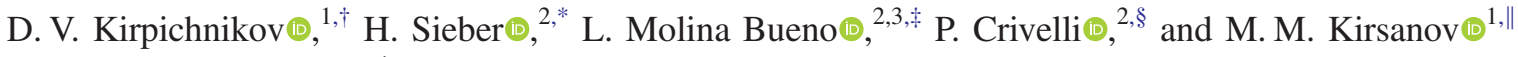 \\ ${ }^{1}$ Institute for Nuclear Research, 117312 Moscow, Russia \\ ${ }^{2}$ ETH Zürich, Institute for Particle Physics and Astrophysics, CH-8093 Zürich, Switzerland \\ ${ }^{3}$ Instituto de Fisica Corpuscular (CSIC/UV), Carrer del Catedrátic José Beltrán Martinez, \\ 2, 46980 Paterna, Valencia
}

(Received 5 August 2021; accepted 10 September 2021; published 14 October 2021)

\begin{abstract}
Vector bosons, such as dark photon $A^{\prime}$ or $Z^{\prime}$, can couple to muons and be produced in the bremsstrahlung reaction $\mu^{-}+N \rightarrow \mu^{-}+N+A^{\prime}\left(Z^{\prime}\right)$. Their possible subsequent invisible decay can be detected in fixed target experiments through missing energy/momentum signature. In such experiments, not only is the energy transfer to $A^{\prime}\left(Z^{\prime}\right)$ important but also the recoil muon angle $\psi_{\mu^{\prime}}$. In this paper, we derive the total and the double differential cross sections involved in this process using the phase space Weizsäcker-Williams and improved Weizsäcker-Williams approximations, as well as using exact-tree-level calculations. As an example, we compare the derived cross sections and resulting signal yields in the NA64 $\mu$ experiment that uses a $160 \mathrm{GeV}$ muon beam at the CERN Super Proton Synchrotron accelerator. We also discuss its impact on the NA64 $\mu$ expected sensitivity to explore the $(g-2)_{\mu}$ anomaly favored region with a $Z^{\prime}$ boson considering $10^{12}$ muons accumulated on target.
\end{abstract}

DOI: 10.1103/PhysRevD.104.076012

\section{INTRODUCTION}

The recently confirmed $4.2 \sigma$ discrepancy in the anomalous magnetic moment measurement of the muon [1] with respect to its theoretical prediction [2]

$$
\Delta a_{\mu} \equiv a_{\mu}(\exp )-a_{\mu}(\mathrm{th})=(251 \pm 59) \times 10^{-11}
$$

remains one of the long standing puzzles in the Standard Model (SM) of particle physics. A minimal extension of the SM able to explain this mismatch consists in the addition of a new weak interaction between the standard matter and a dark sector. The existence of dark sectors is also strongly motivated as a framework to explain the origin of dark matter as a thermal freeze-out relic, a mechanism similar to the one of the weakly interacting massive particles, but in a broader and lower mass range [3]. In particular, the $L_{\mu}-L_{\tau}$ models based on the existence of a new light vector boson

\footnotetext{
* Corresponding author. henri.hugo.sieber@cern.ch

tkirpich@ms2.inr.ac.ru

‡laura.molina.bueno@cern.ch

\$paolo.crivelli@cern.ch

mikhail.kirsanov@cern.ch
}

Published by the American Physical Society under the terms of the Creative Commons Attribution 4.0 International license. Further distribution of this work must maintain attribution to the author(s) and the published article's title, journal citation, and DOI. Funded by SCOAP ${ }^{3}$.
$\left(Z^{\prime}\right)$, which predominantly couples to the second and third generation of leptons, are theoretically well motivated as they are an anomaly-free extension of the gauge sector. The interaction between $Z^{\prime}$ and the $L_{\mu}-L_{\tau}$ current is given by the following term [4]:

$$
L_{Z^{\prime}}=g^{\prime}\left(\bar{\mu} \gamma_{\nu} \mu+\bar{\nu}_{\mu L} \gamma_{\nu} \nu_{\mu L}-\bar{\tau} \gamma_{\nu} \tau-\bar{\nu}_{\tau L} \gamma_{\nu} \nu_{\tau L}\right) Z^{\prime \nu}
$$

This interaction can give via loop effects the required additional contribution to explain the discrepancy between the theoretical and experimental value of the muon magnetic moment [4-9]:

$$
\Delta a_{\mu}^{Z^{\prime}}=\frac{g^{\prime 2}}{4 \pi^{2}} \int_{0}^{1} d x \frac{x^{2}(1-x)}{x^{2}+(1-x) m_{Z^{\prime}}^{2} / m_{\mu}^{2}}
$$

for couplings $g^{\prime} \sim 10^{-3}-10^{-5}$ and $Z^{\prime}$ masses in the $\mathrm{MeV}-\mathrm{GeV}$ range.

If such a boson exists, it can be produced in the muon nuclear bremsstrahlung process $\mu^{-}+N \rightarrow \mu^{-}+N+Z^{\prime}$ when a high energy muon beam impinges on a target. In this paper, we focus on the calculation of this production mechanism but the same results can be directly applied to the $A^{\prime}$ bremsstrahlung $\left(A^{\prime}\right.$-strahlung) production from an initial muon beam. In order to illustrate the impact of our results, we consider the NA64 $\mu$ experiment [10], which aims at exploring dark sector particles weakly coupled to muons. A similar experiment, $M^{3}$, is also under consideration at Fermilab [11]. 
Typically, the $Z$ is expected to be produced in the bremsstrahlung reaction of a $160 \mathrm{GeV}$ muon beam impinging on an electromagnetic calorimeter acting as an active target. A fraction of the primary beam energy is carried away by the scattered muon, which momentum is measured by a set of trackers after being deflected by a magnet located downstream the target. In this work, we derive the cross sections involved in the production process and estimate its impact on the signal yield using the GEANT4-based [12] dark matter package DMG4 [13], in which we implemented the simulation of $Z$ according to the derived cross sections. We compare the results of analytical calculations using phase space approximations such as Weizsäcker-Williams (WW) and improved Weizsäcker-Williams (IWW) with exact-tree-level (ETL) calculations. We also derive a new analytical expression to calculate the photon flux in the WW approximation. Finally, we study the differential cross sections as a function of the recoil muon and $Z^{\prime}$ angles.

This paper is organized as follows. In Sec. II, we calculate at ETL the total cross section for $Z^{\prime}$ production. In Sec. III, we discuss the differential cross sections for the angle and energy fraction of the emitted particle $Z^{\prime}$ for both WW and IWW approximations. In Sec. III, we also discuss the zero mass limit $m_{Z^{\prime}} \rightarrow 0$ of the corresponding cross sections and show that WW and ETL reproduce the bremsstrahlung spectrum of muons on nucleus. In Sec. IV, we derive the differential cross sections for the angle and energy fraction of the deflected muon for both WW and IWW approximations. Finally, in Sec. V, we evaluate the impact of the cross section calculations on the projected $(g-2)_{\mu}$ sensitivities for NA64 ${ }_{\mu}$ experiment.

\section{THE EXACT TREE-LEVEL CALCULATION}

In this section, we discuss the derivation of the $Z^{\prime}$-boson exact-tree-level production cross section, in particular, we follow the notations of Refs. $[14,15]$. Let us consider the kinematic variables of the process

$$
\mu^{-}(p)+N\left(P_{i}\right) \rightarrow \mu^{-}\left(p^{\prime}\right)+N\left(P_{f}\right)+Z^{\prime}(k),
$$

where $p=\left(E_{\mu}, \mathbf{p}\right)$ is the four-momentum of the incoming muon, $P_{i}=(M, 0)$ designates the nucleus fourmomentum in the laboratory frame, and $P_{f}=\left(P_{f}^{0}, \mathbf{P}_{f}\right)$ is the final state of the nucleus. The $Z^{\prime}$-boson momentum is $k=\left(E_{Z^{\prime}}, \mathbf{k}\right)$ and $p^{\prime}=\left(E_{\mu^{\prime}}, \mathbf{p}^{\prime}\right)$ is the momentum of the scattered muon. It is instructive to carry out the calculation in the geometrical frame where the three-vector $\mathbf{V}=\mathbf{p}-\mathbf{k}$ is parallel to $z$ axis and the three-vector $\mathbf{k}$ is in the $x z$ plane. We define the four-momentum transfer to the nucleus as $q=P_{i}-P_{f}$. In that frame the polar and axial angles of $\mathbf{q}$ are denoted by $\theta_{q}$ and $\phi_{q}$, respectively. We use minus metric $\eta_{\mu \nu}=\operatorname{diag}(+,-,-,-)$, in contrast to the authors of Ref. [14], which implies that the virtuality of the photon is $t=-q^{2}=|\mathbf{q}|^{2}-q_{0}^{2}>0$.
After some algebraic manipulations one obtains the following expressions

$$
\begin{gathered}
\cos \theta_{q}=-\frac{|\mathbf{V}|^{2}+|\mathbf{q}|^{2}+m_{\mu}^{2}-\left(E_{\mu}+q_{0}-E_{Z^{\prime}}\right)^{2}}{2|\mathbf{V}||\mathbf{q}|}, \\
q_{0}=-\frac{t}{2 M}, \quad|\mathbf{q}|=\sqrt{\frac{t^{2}}{4 M^{2}}+t .}
\end{gathered}
$$

We assume that the nucleus has zero spin [14,16-18], as a result the photon-nucleus vertex is given by

$$
i e F(t)\left(P_{i}+P_{f}\right)_{\mu} \equiv i e F(t) \mathcal{P}_{\mu},
$$

where the squared elastic form factor is

$$
F^{2}(t) \equiv G_{2}^{e l}(t) \simeq Z^{2}\left(\frac{a^{2} t}{1+a^{2} t}\right)^{2}\left(\frac{1}{1+t / d}\right)^{2},
$$

with $a=111 Z^{-1 / 3} / m_{e}$ and $d=0.164 \mathrm{GeV}^{2} A^{-2 / 3}$. The contribution to the cross section associated with squared inelastic form factor is proportional to $Z$, therefore for heavy nucleus $Z \sim \mathcal{O}(100)$ that term is negligible. In the case of NA64 $\mu$, the active target is made of lead $(A=$ $207, Z=82$ ) being relevant only to the elastic form factors giving the following typical momenta transfer associated with screening effects and nucleus size, respectively,

$$
\begin{aligned}
& \sqrt{t_{a}}=1 / a \simeq 2.0 \times 10^{-5} \mathrm{GeV}, \\
& \sqrt{t_{d}}=\sqrt{d} \simeq 6.9 \times 10^{-2} \mathrm{GeV}
\end{aligned}
$$

We define the energy fraction of the $Z^{\prime}$ boson as $x=E_{Z^{\prime}} / E_{\mu}$ and the angle between $\mathbf{k}$ and $\mathbf{p}$ as $\theta_{Z^{\prime}}$. One can express the differential cross section in the following form [14]

$$
\begin{aligned}
\frac{d \sigma}{d x d \cos \theta_{Z^{\prime}}}= & \frac{\epsilon^{2} \alpha^{3}|\mathbf{k}| E_{\mu}}{|\mathbf{p}||\mathbf{k}-\mathbf{p}|} \cdot \int_{t_{\min }}^{t_{\max }} \frac{d t}{t^{2}} G_{2}^{e l}(t) \\
& \cdot \int_{0}^{2 \pi} \frac{d \phi_{q}}{2 \pi} \frac{\left|\mathcal{A}_{Z^{\prime}}^{2 \rightarrow 3}\right|^{2}}{8 M^{2}}
\end{aligned}
$$

where $\epsilon$ is $Z^{\prime}$ coupling to muons, which is related to $g^{\prime}$ as follows $\epsilon=g^{\prime} / \sqrt{4 \pi \alpha}$, here $\alpha=1 / 137$ is fine-structure constant, $t_{\min }$ and $t_{\max }$ are the values of minimum and maximum momentum transfer, respectively. The quantities for $t_{\min }$ and $t_{\max }$ are derived explicitly in Ref. [14]. The production amplitude squared for vector dark boson such as $Z^{\prime}$ is calculated by using FeynCalc tools $[19,20]$ for the Wolfram Mathematica package [21] 


$$
\begin{aligned}
\left|\mathcal{A}_{Z^{\prime}}^{2 \rightarrow 3}\right|^{2}= & \frac{2}{\tilde{s}^{2} \tilde{u}^{2}}\left(+\tilde{s} \tilde{u}\left\{\mathcal { P } ^ { 2 } \left[(\tilde{s}+t)^{2}\right.\right.\right. \\
& \left.\left.+(\tilde{u}+t)^{2}\right]-4 t\left[(\mathcal{P} \cdot p)^{2}+\left(\mathcal{P} \cdot p^{\prime}\right)^{2}\right]\right\} \\
& +2 m_{\mu}^{2}\left\{\mathcal{P}^{2} t(\tilde{s}+\tilde{u})^{2}-4[(\mathcal{P} \cdot p) \tilde{u}\right. \\
& \left.\left.+\left(\mathcal{P} \cdot p^{\prime}\right) \tilde{s}\right]^{2}\right\}+m_{Z^{\prime}}^{2}\left\{\mathcal{P}^{2} t(\tilde{s}-\tilde{u})^{2}\right. \\
& \left.\left.-4\left[(\mathcal{P} \cdot p) \tilde{u}+\left(\mathcal{P} \cdot p^{\prime}\right) \tilde{s}\right]^{2}\right\}\right),
\end{aligned}
$$

where the Mandelstam variables and relevant dot products are

$$
\begin{aligned}
\tilde{s} & =\left(p^{\prime}+k\right)^{2}-m_{\mu}^{2}=2\left(p^{\prime} \cdot k\right)+m_{Z^{\prime}}^{2}, \\
\tilde{u} & =(p-k)^{2}-m_{\mu}^{2}=-2(p \cdot k)+m_{Z^{\prime}}^{2}, \\
\mathcal{P}^{2} & =4 M^{2}+t, \quad \mathcal{P} \cdot p=2 M E_{\mu}-(\tilde{s}+t) / 2, \\
\mathcal{P} \cdot p^{\prime} & =2 M\left(E_{\mu}-E_{Z^{\prime}}\right)+(\tilde{u}-t) / 2 .
\end{aligned}
$$

The resulting amplitude squared (11) coincides with the one given in Ref. [14] for the case considered here, replacing the incident electron with a muon.

\section{THE APPROXIMATIONS FOR THE $Z^{\prime}$ EMISSION CROSS SECTIONS}

To calculate the differential $Z^{\prime}$ emission cross sections in the muon-nuclei interactions we can use the so-called Weizsäcker-Williams approximation by assuming that the energy of the initial particle is much higher than $m_{\mu}$ and $m_{Z^{\prime}}$. In this case, the flux of generated virtual photons can be considered as a plane wave and be approximated by a real photon. This approximation allows thus to reduce the phase space of a $\mu(p)+N\left(\mathcal{P}_{i}\right) \rightarrow Z^{\prime}(k)+\mu\left(p^{\prime}\right)+N\left(\mathcal{P}_{f}\right)$ process to a Compton-like $\mu(p)+\gamma(q) \rightarrow Z^{\prime}(k)+\mu\left(p^{\prime}\right)$ process $[6,15,22]$. In particular, following the procedure described in Appendix D of [22], which generalizes the classical WW one photon exchange process, we write down the following expression:

$$
\begin{aligned}
& \left.\frac{d \sigma\left(p+\mathcal{P}_{i} \rightarrow k+p^{\prime}+\mathcal{P}_{f}\right)}{d(p k) d\left(k \mathcal{P}_{i}\right)}\right|_{\mathrm{WW}} \\
& =\left.\frac{\alpha \chi}{\pi\left(p^{\prime} \mathcal{P}_{i}\right)} \cdot \frac{d \sigma\left(p+q \rightarrow k+p^{\prime}\right)}{d(p k)}\right|_{t=t_{\min }} .
\end{aligned}
$$

For ultrarelativistic incident muons we take into account that in the laboratory frame $d\left(k \mathcal{P}_{i}\right)=M d E_{Z^{\prime}},\left(p^{\prime} \mathcal{P}_{i}\right)=M E_{\mu^{\prime}}$, $d(p k) \simeq-|\mathbf{p}||\mathbf{k}| d \cos \theta_{Z^{\prime}}, \quad|\mathbf{k}|=\sqrt{E_{Z^{\prime}}^{2}-m_{Z^{\prime}}^{2}}$ and $E_{\mu^{\prime}} \simeq$ $E_{\mu}-E_{Z^{\prime}}$. The WW approach implies that the virtuality $t$ has its minimum $t_{\min }$ when $\mathbf{q}$ is collinear with $\mathbf{k}-\mathbf{p}$. For the sake of simplicity we denote the cross section on the left side of Eq. (14) as $d \sigma_{2 \rightarrow 3}$ and in the right side as $d \sigma_{2 \rightarrow 2}$. We obtain the following expression for the double differential cross section: $\left.\left.\frac{d \sigma_{2 \rightarrow 3}}{d x d \cos \theta_{Z^{\prime}}}\right|_{\mathrm{WW}} \simeq \frac{\alpha \chi}{\pi(1-x)} \cdot E_{\mu}^{2} x \beta_{Z^{\prime}} \cdot \frac{d \sigma_{2 \rightarrow 2}}{d(p k)}\right|_{t=t_{\min }}$,

where $\beta_{Z^{\prime}}=\sqrt{1-m_{Z^{\prime}}^{2} /\left(x^{2} E_{\mu}^{2}\right)}$. It is worth mentioning that the authors of Ref. [23] made a typo in the $\beta_{Z^{\prime}}$ definition. We take into account the $x$ dependence in $\beta_{Z^{\prime}}$ as in Ref. [14], which plays an important role for $x \ll 1$. The effective photon flux $\chi$ in the WW approach is defined by

$$
\chi^{\mathrm{WW}}=\int_{t_{\min }}^{t_{\max }} d t \frac{t-t_{\min }}{t^{2}} F^{2}(t)
$$

where $t_{\min } \approx U^{2}\left(x, \theta_{Z^{\prime}}\right) /\left(4 E_{\mu}^{2}(1-x)^{2}\right)$ and $t_{\max }=m_{Z^{\prime}}^{2}+$ $m_{\mu}^{2}$ are the minimum and maximum squared momentum transfer to the nucleus. The expression for $t_{\min }$ is derived below [for details, see, e.g., Eq. (21) and its discussion] and the function $U\left(x, \theta_{Z^{\prime}}\right)$ is defined by

$$
\begin{aligned}
U \equiv & 2\left(E_{Z^{\prime}} E_{\mu}-|\mathbf{k}||\mathbf{p}| \cos \theta_{Z^{\prime}}\right)-m_{Z^{\prime}}^{2} \simeq E_{\mu}^{2} \theta_{Z^{\prime}}^{2} x \\
& +m_{Z^{\prime}}^{2}(1-x) / x+m_{\mu}^{2} x .
\end{aligned}
$$

In Eq. (17) we keep only leading terms in $m_{Z^{\prime}}^{2} / E_{Z^{\prime}}^{2}, m_{\mu}^{2} / E_{\mu^{\prime}}^{2}$, and $\theta_{Z^{\prime}}^{2}$. Considering the subsequent identities for the momentum squared

$$
M^{2}=\left(\mathcal{P}_{i}-q\right)^{2}, \quad q^{2}=\left(\mathcal{P}_{i}-\mathcal{P}_{f}\right)^{2}=2 M q_{0},
$$

implies that the expression for the typical energy transferred to the nucleus can be expressed as

$$
q_{0} \simeq-|\mathbf{q}|^{2} /(2 M) .
$$

This value is negligible and will be ignored in the calculation below. One can easily obtain the expression $|\mathbf{k}-\mathbf{p}| \simeq$ $E_{\mu}(1-x)$ by taking into account the approximation discussed above. The next step is to consider the kinematic identity for the four-momentum squared of the outgoing muon:

$$
\left(p^{\prime}\right)^{2}=(q-k+p)^{2} \equiv m_{\mu}^{2}
$$

For $|\mathbf{q}|^{2} \ll U$ and $|\mathbf{q}|^{2} \ll|\mathbf{k}-\mathbf{p}|^{2}$ Eq. (20) implies the relation between the absolute value of nucleus momentum transfer and other kinematic variables

$$
|\mathbf{q}| \simeq U /(2|\mathbf{k}-\mathbf{p}|) \simeq U /\left(2 E_{\mu}(1-x)\right) .
$$

Note that in Eq. (21) we take into account that the vectors $\mathbf{q}$ and $\mathbf{k}-\mathbf{p}$ are collinear. Therefore, for WW approximation we get the expression for the minimum nucleus momentum transfer, $t_{\min } \simeq|\mathbf{q}|^{2}$. 
The final ingredient to obtain the differential cross section is the $\sigma_{2 \rightarrow 2}$ calculation. The differential cross section in the Lorentz invariant notations [23] is

$$
\frac{d \sigma_{2 \rightarrow 2}}{d(p k)}=\frac{d \sigma_{2 \rightarrow 2}}{d\left(p p^{\prime}\right)}=\frac{2 \pi \alpha^{2} \epsilon^{2}}{\tilde{s}} \cdot\left|\mathcal{A}_{2 \rightarrow 2}^{Z^{\prime}}\right|^{2},
$$

where [14]

$$
\begin{aligned}
\left|\mathcal{A}_{2 \rightarrow 2}^{Z^{\prime}}\right|^{2}= & -2 \frac{\tilde{s}}{\tilde{u}}-2 \frac{\tilde{u}}{\tilde{s}}+4\left(m_{Z^{\prime}}^{2}\right. \\
& \left.+2 m_{\mu}^{2}\right)\left[\left(\frac{\tilde{s}+\tilde{u}}{\tilde{s} \tilde{u}}\right)^{2} m_{\mu}^{2}-\frac{t_{2}}{\tilde{s} \tilde{u}}\right],
\end{aligned}
$$

where the Mandelstam variables are defined as follows

$$
\begin{aligned}
\tilde{u} & =(p-k)^{2}-m_{\mu}^{2}, \\
\tilde{s} & =\left(p^{\prime}+k\right)^{2}-m_{\mu}^{2}, \\
t_{2} & =\left(p-p^{\prime}\right)^{2} .
\end{aligned}
$$

We note that the authors of Ref. [23] dropped terms in (23) that are associated with a squared incident particle mass (the electron mass term in Ref. [23]). As explained in [14], dropping these terms will lead to an underestimation of the experiment sensitivity region. Moreover, when the incident particle is a muon, one can not drop the corresponding terms [14] since the underestimation of the available parameter space will be significant as soon as $m_{Z^{\prime}} \lesssim m_{\mu}$. Finally by using the relation for the scalar product $(q k) \simeq$ $U x /(2(1-x))$ and the approximate expression $\tilde{s}+t_{2}+$ $\tilde{u} \simeq m_{Z^{\prime}}^{2}$ one can easily get

$$
\begin{aligned}
\tilde{u} & \simeq-U, \quad \tilde{s} \simeq U /(1-x), \\
t_{2} & \simeq-x U /(1-x)+m_{Z^{\prime}}^{2} .
\end{aligned}
$$

We use Eqs. (25) below in Secs. III A and III B to derive the double differential cross section in both WW and IWW approach.

\section{A. Cross section in WW approximation}

The photon flux can be integrated numerically according to Eq. (16). However, it is instructive and less computational demanding to develop an analytical expression for this equation by using the specific form of elastic form factor, Eq. (7). In this case, one can get the following analytical expression for the effective photon flux

$$
\chi=Z^{2}\left[\tilde{\chi}\left(t_{\max }\right)-\tilde{\chi}\left(t_{\min }\right)\right],
$$

where

$$
\begin{aligned}
\tilde{\chi}(t)= & \frac{t_{d}^{2}}{\left(t_{a}-t_{d}\right)^{3}}\left[\frac{\left(t_{a}-t_{d}\right)\left(t_{a}+t_{\min }\right)}{t+t_{a}}\right. \\
& +\frac{\left(t_{a}-t_{d}\right)\left(t_{d}+t_{\min }\right)}{t+t_{d}} \\
& \left.+\left(t_{a}+t_{d}+2 t_{\min }\right) \log \left(\frac{t+t_{d}}{t+t_{a}}\right)\right],
\end{aligned}
$$

which is valid for both WW and IWW approaches. Here $t_{a}$ and $t_{d}$ are defined by Eqs. (8) and (9), respectively. However, in this subsection we specify the photon flux for the case of double differential WW cross section, which simply implies that $t_{\min } \approx U^{2}\left(x, \theta_{Z^{\prime}}\right) /\left(4 E_{\mu}^{2}(1-x)^{2}\right)$. In particular, one has the following expression for the WW cross section of $Z^{\prime}$ boson

$$
\left(\frac{d \sigma_{2 \rightarrow 3}}{d x d \cos \theta_{Z^{\prime}}}\right)_{\mathrm{WW}}=2 \epsilon^{2} \alpha^{3} \sqrt{x^{2}-\frac{m_{Z^{\prime}}^{2}}{E_{\mu}^{2}}} E_{\mu}^{2}(1-x) \frac{\chi^{\mathrm{WW}}}{\tilde{u}^{2}}\left|\mathcal{A}_{2 \rightarrow 2}^{Z^{\prime}}\right|^{2} .
$$

By substituting Eqs. (17) and (25) into Eq. (23), the amplitude squared can be rewritten as [14]

$$
\begin{aligned}
\left|\mathcal{A}_{2 \rightarrow 2}^{Z^{\prime}}\right|^{2}= & 2 \frac{2-2 x+x^{2}}{1-x} \\
& +4\left(m_{Z^{\prime}}^{2}+2 m_{\mu}^{2}\right) \frac{\tilde{u} x+m_{Z^{\prime}}^{2}(1-x)+m_{\mu}^{2} x^{2}}{\tilde{u}^{2}} .
\end{aligned}
$$

Now let us specify the phase space for the produced $Z^{\prime}$. In particular, Eq. (28) implies that $x_{\min } \equiv m_{Z^{\prime}} / E_{\mu} \lesssim x$. The nuclear transfer energy is given by Eq. (19) so that it can be neglected for the large $Z^{\prime}$ energy fraction bound $x \lesssim 1$. Therefore one can immediately get the following upper bound on $x \lesssim x_{\max } \equiv 1-m_{\mu} / E_{\mu}$.

\section{B. Calculations using IWW approximation}

In the so-called improved WW approach the dependence of $t_{\min }$ on $x$ and $\theta_{Z^{\prime}}$ in the flux derivation [Eq. (16)] is omitted to simplify calculations, which is important in Monte Carlo (MC) simulations, such that $t_{\min }^{\mathrm{IWW}} \simeq m_{Z^{\prime}}^{4} /\left(4 E_{\mu}^{2}\right)$. This means however that the IWW approach is less accurate [14].

We compute the differential cross section using the IWW approximation to compare with the approach developed in the previous section. The integration over $\theta_{Z^{\prime}}$ in this case can be performed analytically [see, e.g., Eq. (30) in Ref. [14] ]. In particular, one has 


$$
\begin{aligned}
& \left(\frac{d \sigma_{2 \rightarrow 3}}{d x}\right)_{\mathrm{IWW}} \\
& =2 \epsilon^{2} \alpha^{3} \chi^{\mathrm{IWW}} \sqrt{x^{2}-\frac{m_{Z^{\prime}}^{2}}{E_{\mu}^{2}}} \\
& \times\left.\left[\frac{m_{\mu}^{2} x\left(-2+2 x+x^{2}\right)-2\left(3-3 x+x^{2}\right) \tilde{u}}{3 x \tilde{u}^{2}}\right]\right|_{\tilde{u}_{\min }} ^{\tilde{u}_{\max }}
\end{aligned}
$$

where $\tilde{u}_{\max }=-m_{Z^{\prime}}^{2} \frac{1-x}{x}-m_{\mu}^{2} x$ and $\tilde{u}_{\min }=-x E_{\mu}^{2}\left(\theta_{Z^{\prime}}^{\max }\right)^{2}-$ $m_{Z^{\prime}}^{2} \frac{1-x}{x}-m_{\mu}^{2} x$. In contrast to Ref. [14], we keep $\tilde{u}_{\text {min }}$ finite to take into account the effects associated with the maximal angle of emitted $Z^{\prime}$. As discussed before, in the IWW approximation $\chi$ is independent of $x$ and $\theta_{Z^{\prime}}$. The resulting IWW cross section has a sharp peak at $x \simeq 1$, as illustrated in Fig. 1. The numerical calculations reveal that this peak is smeared in WW approach due to the fact that $\chi$ is a function of $x$ and $\theta_{Z^{\prime}}$ (see the green line in the right plot of Fig. 1).

The comparison between the differential cross section as a function of $x$ calculated using the three approaches for

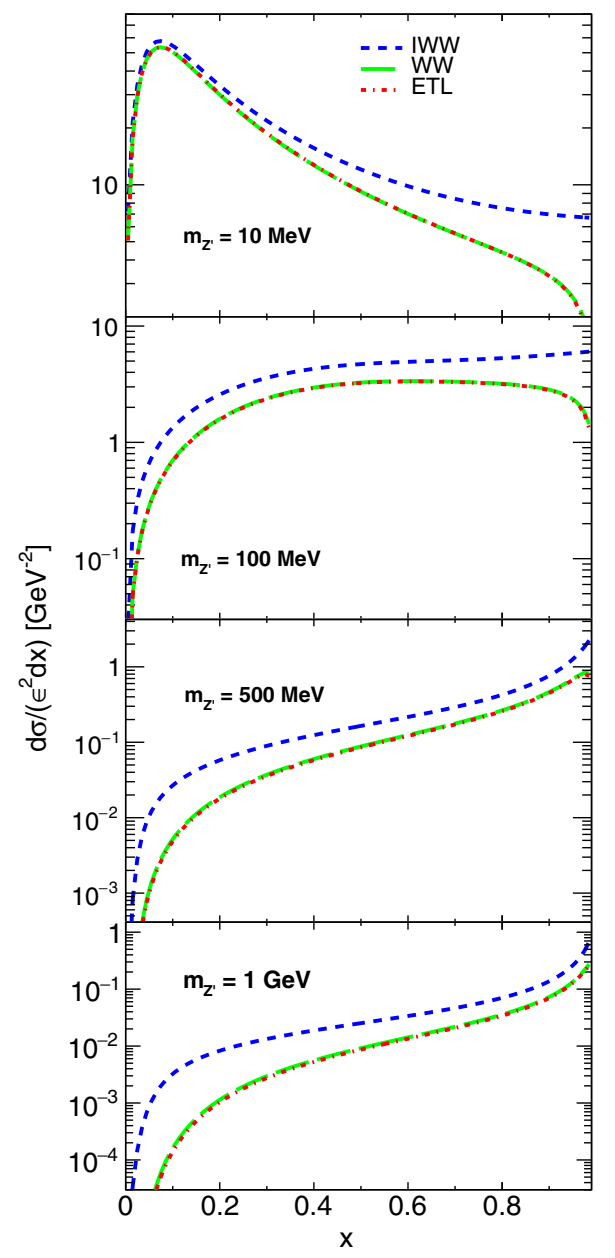

different $Z^{\prime}$ masses is shown in Fig. 1. The muon energy has been set to $160 \mathrm{GeV}$ as expected in $\mathrm{NA} 64 \mu$ experiment. We note that the typical angle of $Z^{\prime}$ emission does not depend on its mass as it scales as $\theta_{Z^{\prime}} \sim m_{\mu} / E_{\mu}$. This can be seen in Fig. 3, left plot. Thus, the differential cross sections [see, e.g., Eqs. (30), (32), and (10)] are numerically integrated using, respectively, adaptive and Monte Carlo procedures [24] up to a maximum $Z^{\prime}$ angle $\theta_{Z^{\prime}}^{\max } \sim 0.1$.

The values obtained for the differential cross section are different depending on the mass because of the dynamic conditions. In the right plots of Fig. 1, the relative difference between the approximations with respect to the exacttree-level calculation is shown. The differences between WW and the exact calculation are below $2 \%$ in the mass region between $10 \mathrm{MeV}$ and $1 \mathrm{GeV}$. Deviations from exact cross sections are significant in the case of IWW approximation, especially in the extreme fractional energy regions, due to the flux simplification.

In Fig. 2 the evolution of the total cross section, calculated with the different approaches, is presented as

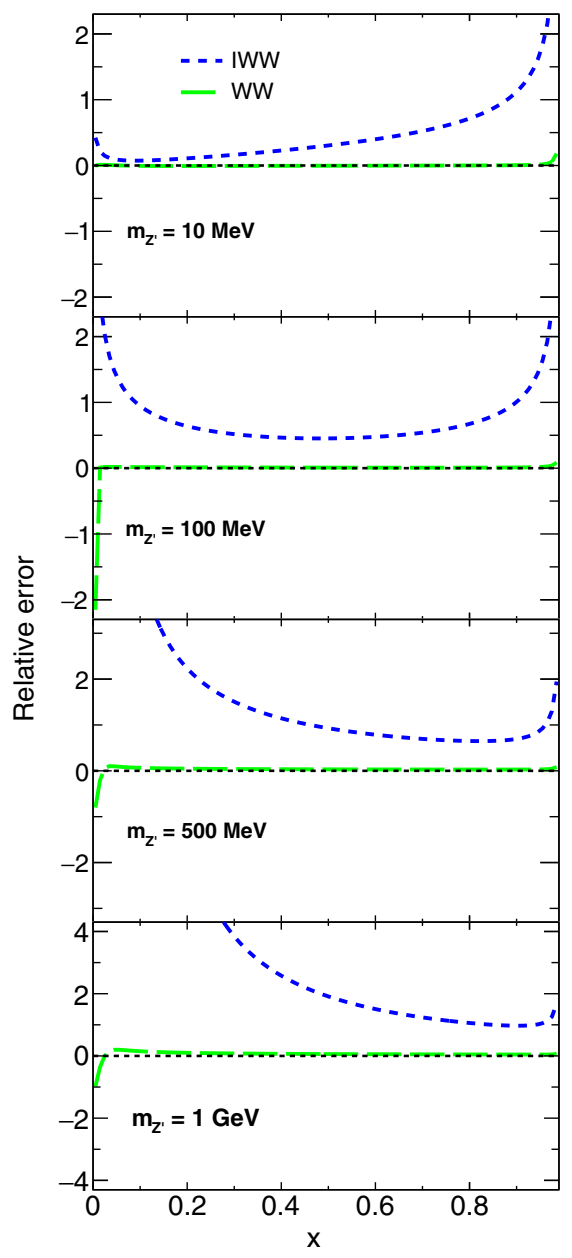

FIG. 1. Left: differential cross section as a function of $x$ for IWW (blue dashed line), WW (green line), and ETL (red dotted line) for different $Z^{\prime}$ masses. The single- and double-differential cross sections [see, e.g., Eqs. (30), (32), and (10)] are numerically integrated using, respectively, adaptive and Monte Carlo procedures [24]. Right: relative error between WW and IWW approximations as a function of $x$ defined as $\left(\mathcal{O}_{\text {approx }}-\mathcal{O}_{\text {exact }}\right) / \mathcal{O}_{\text {exact }}$ (similar approach than the one used in [14]). 

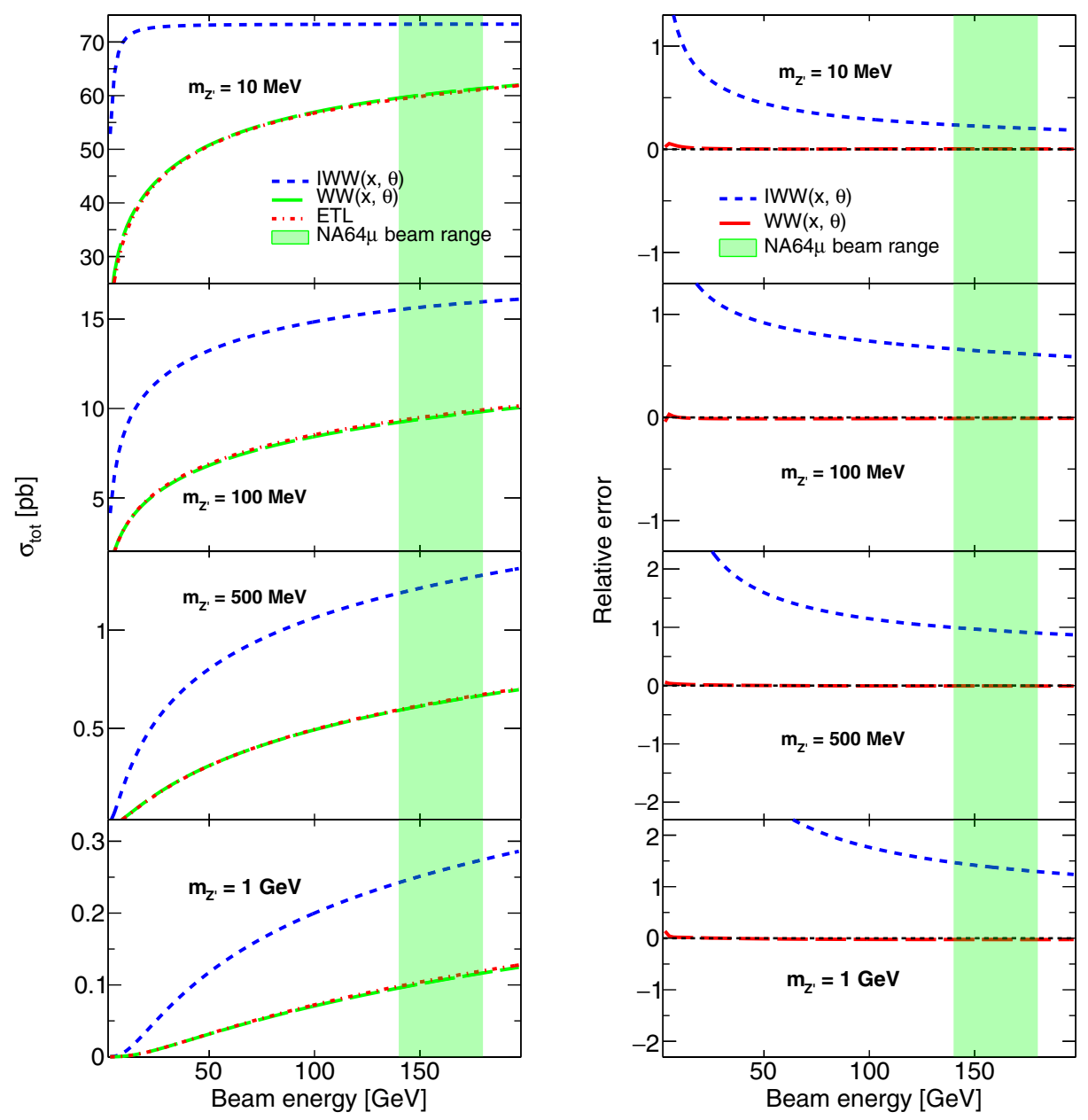

FIG. 2. Left: total cross section as a function of the beam energy for IWW (blue dashed line), WW (green line) expressions, and ETL (red dotted line) integrated over $x, \theta$. Right: relative error between WW, IWW approximations, and ETL as a function of the beam energy expressed as $\left(\mathcal{O}_{\text {approx }}-\mathcal{O}_{\text {exact }}\right) / \mathcal{O}_{\text {exact }}$. The typical NA64 $\mu$ beam energy range is shown as a green band.

a function of the initial muon energy for different $Z^{\prime}$ masses. The WW approximation reproduces the ETL cross section with a relative difference below $2 \%$ for the whole mass range. For illustration, the energy region interesting for the NA64 $\mu$ experiment is highlighted in green. In this region, the relative differences between IWW and ETL (Sec. II) are smaller than in the low energy region, where they tend to diverge, when the muon energy approaches the $Z^{\prime}$ mass.

\section{Zero mass limit for WW approach}

An independent cross-check to validate our calculations, is to consider the case of massless $Z^{\prime},\left(m_{Z^{\prime}} \rightarrow 0\right)$, since this should reproduce the muon nuclear bremsstrahlung $\mu N \rightarrow$ $\mu N \gamma$ cross section [25]. We have used a simplified analytical expression of the flux for these calculations valid for the parameter space of interest $t_{\max } \gg t_{d} \gg t_{\min }$ and $t_{d} \gg t_{a}$ with logarithmic accuracy:

$$
\frac{\chi}{Z^{2}} \simeq \log \left(\frac{t_{d}}{t_{\min }+t_{a}}\right)-2 .
$$

We note that this expression is no longer valid as $t_{\min } \simeq t_{d}$, which implies large masses $\mathcal{O}(1) \mathrm{GeV}$, low energies $E_{\mu} \lesssim$ $10 \mathrm{GeV}$ and $x \simeq 1$. However, for the interesting NA64 $\mu$ parameter space, one can use the approximated formula for the calculation of the analytical differential cross section $(d \sigma / d x)_{\mathrm{Ww}}$.

In particular, the numerical calculation reveals that for $m_{A^{\prime}} \lesssim 500 \mathrm{MeV}$ and $E_{\mu} \simeq 150-160 \mathrm{GeV}$ the discrepancy between the cross sections calculated with (31) and (26) is well below $1 \%$. We derive the approximate analytical formula for $Z^{\prime}$ production cross section using WW approximation for this particular case considering only an elastic nuclear form factor. The first step is to integrate out $\theta_{Z^{\prime}}$ in Eq. (28). We obtain 

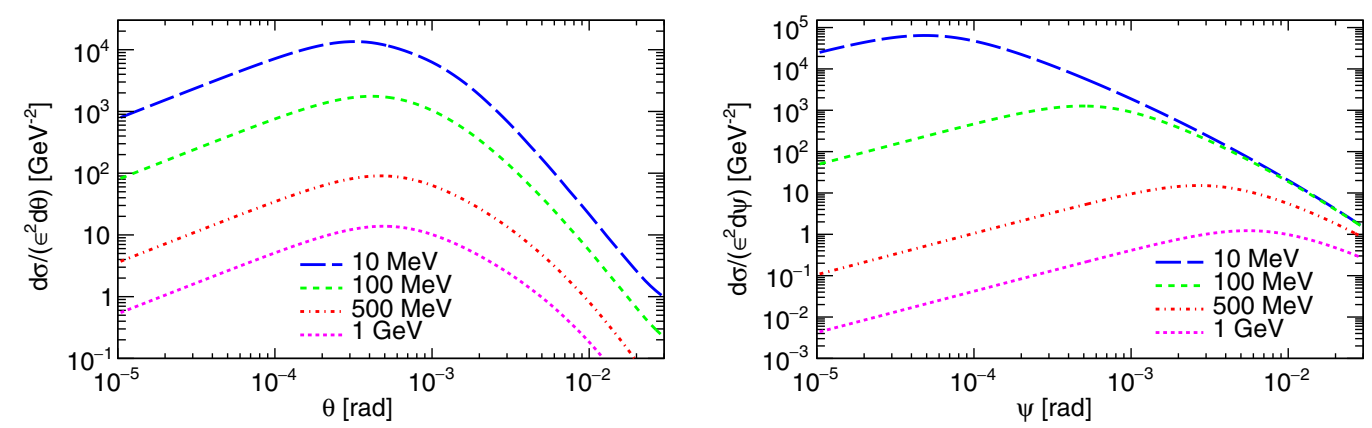

FIG. 3. Differential cross sections in the WW approach as a function of $\theta$ (left) and $\psi$ (right), peaking, respectively, around $\theta_{Z^{\prime}} \simeq$ $m_{\mu} / E_{\mu}$ and $\psi_{\mu^{\prime}} \simeq m_{Z^{\prime}} / E_{\mu}$.

$$
\left(\frac{d \sigma}{d x}\right)_{\mathrm{WW}}=\epsilon^{2} \alpha^{3} \sqrt{x^{2}-m_{Z^{\prime}}^{2} / E_{\mu}^{2}} \frac{(1-x)}{x} \int_{\tilde{u}_{\min }}^{\tilde{u}_{\max }} d \tilde{u} \frac{\left|\mathcal{A}_{2 \rightarrow 2}^{Z^{\prime}}\right|^{2}}{\tilde{u}^{2}} \chi^{\mathrm{WW}} .
$$

Finally, we get

$$
\left(\frac{d \sigma}{d x}\right)_{\mathrm{WW}}=\epsilon^{2} \alpha^{3} Z^{2} \sqrt{x^{2}-m_{Z^{\prime}}^{2} / E_{\mu}^{2}} \frac{(1-x)}{x} \times\left\{2 \frac{2-2 x+x^{2}}{1-x} I_{2}+4\left(m_{Z^{\prime}}^{2}+2 m_{\mu}^{2}\right)\left[x I_{3}+\left(m_{Z^{\prime}}^{2}(1-x)+m_{\mu}^{2} x^{2}\right) I_{4}\right]\right\} .
$$

where the auxiliary integrals are

$I_{n}=\int_{\tilde{u}_{\min }}^{\tilde{u}_{\max }} d \tilde{u} \frac{1}{\tilde{u}^{n}} \log \left[\frac{\beta_{d}^{2}}{\tilde{u}^{2}+\beta_{a}^{2}}\right], \quad$ for $n=2,3,4$.

In this expression $\beta_{d}^{2}=4 E_{\mu}^{2}(1-x)^{2} t_{d} / e^{2}$, being $e \simeq$ 2.71828 the Euler's number, $\beta_{a}^{2}=4 E_{\mu}^{2}(1-x)^{2} t_{a}$ and $\tilde{u}_{\text {min }}=-x\left(\theta_{Z^{\prime}}^{\max }\right)^{2} E_{\mu}^{2}-m_{Z^{\prime}}^{2} \frac{1-x}{x}-m_{\mu}^{2} x$. The resulting integrals for different $n$ values are

$$
I_{2}=\left.\left[-\frac{1}{\tilde{u}} \log \left(\frac{\beta_{d}^{2}}{\beta_{a}^{2}+\tilde{u}^{2}}\right)-\frac{2}{\beta_{a}} \arctan \left(\frac{\tilde{u}}{\beta_{a}}\right)\right]\right|_{\tilde{u}_{\min }} ^{\tilde{u}_{\max }}
$$

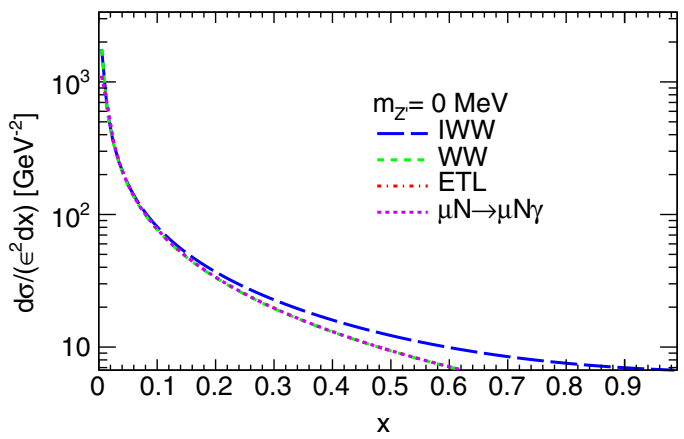

$$
I_{3}=\left.\left[-\frac{1}{2 \tilde{u}^{2}} \log \left(\frac{\beta_{d}^{2}}{\beta_{a}^{2}+\tilde{u}^{2}}\right)+\frac{1}{2 \beta_{a}^{2}} \log \left(\frac{\beta_{a}^{2}+\tilde{u}^{2}}{\tilde{u}^{2}}\right)\right]\right|_{\tilde{u}_{\min }} ^{\tilde{u}_{\max }}
$$

$$
I_{4}=\left.\left[-\frac{1}{3 \tilde{u}^{3}} \log \left(\frac{\beta_{d}^{2}}{\beta_{a}^{2}+\tilde{u}^{2}}\right)+\frac{2}{3 \tilde{u} \beta_{a}^{2}}+\frac{2}{3 \beta_{a}^{3}} \arctan \left(\frac{\tilde{u}}{\beta_{a}}\right)\right]\right|_{\tilde{u}_{\min }} ^{\tilde{u}_{\max }}
$$

In Fig. 4 the differential cross sections for the three approaches in the low $Z^{\prime}$ mass region are compared to the cross section obtained for the QED muon bremsstrahlung process (purple dotted line). The shape of the distributions

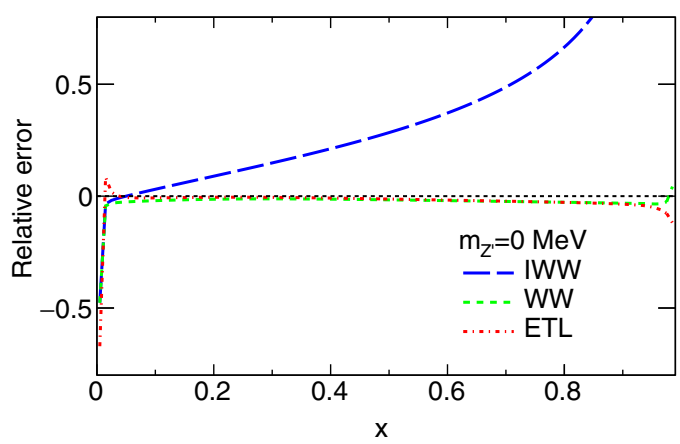

FIG. 4. Left: differential cross sections as a function of $Z^{\prime}$ energy fraction $x$ calculated at ETL and in WW and IWW approaches for $m_{Z^{\prime}}=0$ and $\epsilon=1$. Muon nuclear bremsstrahlung $\mu N \rightarrow \mu N \gamma$ cross section [26] is shown as a purple dotted line. One can see that as soon as $m_{Z^{\prime}} \rightarrow 0$ the shape of the muon bremsstrahlung $\mu N \rightarrow \mu N \gamma$ is reproduced by $\mu N \rightarrow \mu N Z^{\prime}$. Right: relative error defined as $\left(\mathcal{O}_{m=0}-\mathcal{O}_{\text {brem }}\right) / \mathcal{O}_{\text {brem. }}$. The angle of $Z^{\prime}$ emission is integrated in the range $\theta_{Z^{\prime}} \in[0,0.1]$. 
is identical, and the relative differences of the WW and ETL distributions with respect to the muon nuclear bremsstrahlung are below $2 \%$.

\section{DIFFERENTIAL CROSS SECTION FOR THE SCATTERED MUON}

For the successful detection of $Z^{\prime}$ in fixed target experiments such as NA64 $\mu$, it is crucial to tag the scattered muon after $Z^{\prime}$ emission. In this section, we focus on calculating the differential cross section as a function of the scattered muon angle $\psi_{\mu^{\prime}}$ to evaluate its possible impact in the expected signal yield. In the WW approximation, the differential cross section for the process $\mu(p)+N\left(\mathcal{P}_{i}\right) \rightarrow Z^{\prime}(k)+\mu\left(p^{\prime}\right)+N\left(\mathcal{P}_{f}\right)$ is

$$
\frac{d \sigma}{d \cos \psi_{\mu^{\prime}} d y}=\frac{\alpha \chi}{\pi} \frac{E_{\mu}^{2} y \beta_{\mu^{\prime}}}{(1-y)} \times\left.\frac{d \sigma_{2 \rightarrow 2}}{d\left(p p^{\prime}\right)}\right|_{t=t_{\min }},
$$

where $y=E_{\mu}^{\prime} / E_{\mu}$ and $\beta_{\mu^{\prime}}=\sqrt{1-m_{\mu}^{2} /\left(E_{\mu}^{\prime 2}\right)}$ are the fraction energy and the velocity of the outgoing muon, respectively. One can obtain the following differential cross section of the Compton-like process $e \gamma \rightarrow e Z^{\prime}$, which is written in Lorentz-invariant form

$$
\left.\frac{d \sigma_{2 \rightarrow 2}}{d\left(p p^{\prime}\right)}\right|_{t=t_{\min }}=\frac{4 \pi \alpha^{2} \epsilon^{2}}{\tilde{s}^{2}} \times\left(-\frac{\tilde{s}}{\tilde{u}}-\frac{\tilde{u}}{\tilde{s}}+2\left(m_{Z^{\prime}}^{2}+2 m_{\mu}^{2}\right)\left[\left(\frac{\tilde{s}+\tilde{u}}{\tilde{s} \tilde{u}}\right)^{2} m_{\mu}^{2}-\frac{t_{2}}{\tilde{s} \tilde{u}}\right]\right) .
$$

The WW approach implies that the cross section (39) is calculated for the minimum value of the virtuality $t_{\min }$, i.e., when $\mathbf{q}$ is collinear with $\mathbf{p}^{\prime}-\mathbf{p}$. The later condition in association with the relation $k^{2} \equiv m_{Z^{\prime}}^{2}=\left(q-\left(p^{\prime}-p\right)\right)^{2}$ yields

$$
2|\mathbf{q}|\left|\mathbf{p}^{\prime}-\mathbf{p}\right|+t_{2} \simeq m_{Z^{\prime}}^{2}
$$

One can write down the relation $\left|\mathbf{p}^{\prime}-\mathbf{p}\right| \simeq E_{\mu}(1-y)$, which is valid as soon as $m_{\mu} \ll E_{\mu}, E_{\mu^{\prime}}$ and $\psi_{\mu^{\prime}} \ll 1$. Therefore it yields

$$
t_{\min }=|\mathbf{q}|^{2}, \quad|\mathbf{q}|=\frac{m_{Z^{\prime}}^{2}-t_{2}}{2 E_{\mu}(1-y)} .
$$

We also use below the following notation for the auxiliary Mandelstam variable $\tilde{t}=m_{Z^{\prime}}^{2}-t_{2}$. The Mandelstam variables $\tilde{s}, \tilde{u}$, and $t_{2}$ can be written then as

$$
\begin{aligned}
\tilde{s} & =\left(p^{\prime}+k\right)^{2}-m_{\mu}^{2} \simeq \tilde{t} /(1-y), \\
\tilde{u} & =\left(p^{\prime}-q\right)^{2}-m_{\mu}^{2} \simeq-2\left(p^{\prime} q\right) \simeq 2\left|\mathbf{p}^{\prime}\right||\mathbf{q}| \cos \theta_{p^{\prime} q} \\
& \simeq-2\left|\mathbf{p}^{\prime}\right||\mathbf{q}| \simeq-y \tilde{t} /(1-y), \\
t_{2} & =-\left[E_{\mu}^{2} \psi_{\mu^{\prime}}^{2} y+m_{\mu}^{2}(1-y) / y+m_{\mu}^{2} y\right]+m_{\mu}^{2} .
\end{aligned}
$$

In the WW approach the effective photon flux $\chi$ in (38) is defined in (16) with $t_{\min }$ to be calculated using (41) and $t_{\max }=m_{Z^{\prime}}^{2}+m_{\mu}^{2}$. Finally, the differential cross section as a function of the muon fractional energy $y$ and the recoil muon angle $\psi_{\mu^{\prime}}$ [Eq. (38)], can be written as follows:

$\frac{d \sigma}{d \cos \psi_{\mu^{\prime}} d y}=8 \alpha^{3} \epsilon^{2} \chi^{\mathrm{WW}} E_{\mu}^{2} \sqrt{y^{2}-m_{\mu}^{2} / E_{\mu}^{2}} \cdot \frac{(1-y)}{\tilde{t}^{2}} \cdot\left[\frac{1}{2 y}+\frac{y}{2}+\left(m_{Z^{\prime}}^{2}+2 m_{\mu}^{2}\right) \frac{(1-y)^{2}}{\tilde{t}^{2} y}\left(m_{\mu}^{2} \frac{(1-y)^{2}}{y}+m_{Z^{\prime}}^{2}-\tilde{t}\right)\right]$.

The next step is to define our integration limits. One can see from Eq. (43) that the minimal muon fractional energy after emission is $y_{\min } \simeq m_{\mu} / E_{\mu}$, when almost all energy of the initial muon is transferred to $Z^{\prime}$. On the other hand, when the muon transfers close to zero energy to $Z^{\prime}$ we have $y_{\max } \simeq 1-m_{Z^{\prime}} / E_{\mu}$.

Figures 5 and 6 show the results of the numerical integration of the double-differential cross section with respect to the muon fractional energy and the recoil angle $\psi_{\mu^{\prime}}$ for different $Z^{\prime}$ masses in WW and IWW approximations. The difference between the two approximations becomes significant for large recoil muon angles and for large $y$ values due to the flux simplification used in IWW approximation described in the previous section. The behavior of the differential cross section as a function of $y$, reproduces what we observe as a function of $x$ taking into account that $y=1-x$ (see Fig. 1).

The total cross sections in the Weizsäcker-Williams approximation for both sets of variables $\left(x, \theta_{Z^{\prime}}\right)$ and $\left(y, \psi_{\mu^{\prime}}\right)$ have been calculated as an independent crosscheck. The results are compared in Fig. 7 as a function of the beam energy. One can see from Fig. 3 that the typical 

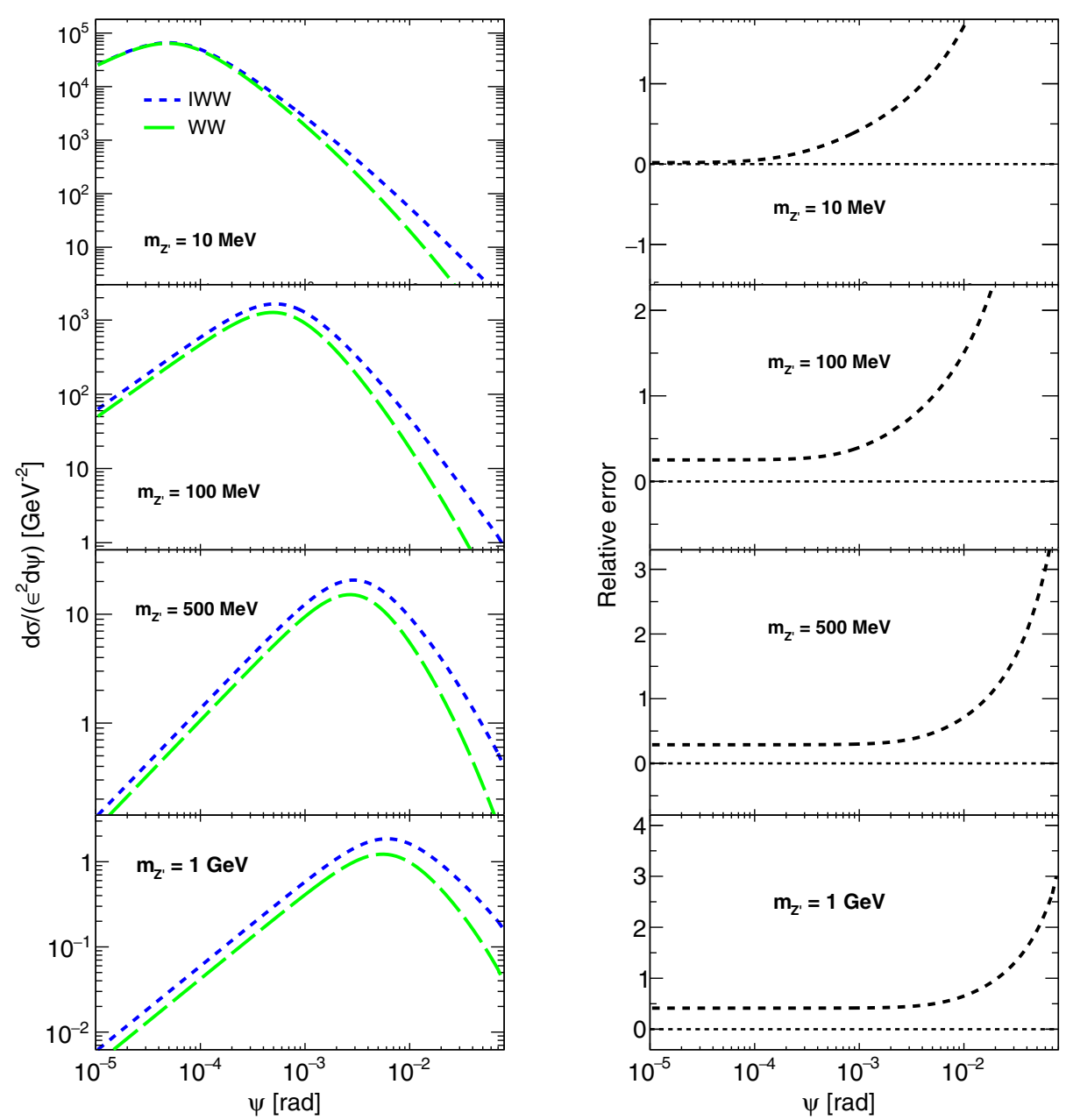

FIG. 5. Left: differential cross section as a function of $\psi_{\mu^{\prime}}$ for IWW (blue dashed line) and WW (green line) for different $Z^{\prime}$ masses. Right: relative error between IWW and WW defined as $\left(\mathcal{O}_{\text {IWW }}-\mathcal{O}_{\mathrm{WW}}\right) / \mathcal{O}_{\mathrm{WW}}$.

angle of $Z^{\prime}$ emission is constant: it depends only on the energy of incoming muon $E_{\mu} \simeq 160 \mathrm{GeV}$ as $\theta_{Z^{\prime}} \simeq m_{\mu} / E_{\mu}$. This implies that the total cross section calculated for $Z^{\prime}$ depends weakly on the angle cut $\theta_{Z^{\prime}}^{\max }$, as long as $\theta_{Z^{\prime}}^{\max } \gg m_{\mu} / E_{\mu} \sim 4 \times 10^{-4}$. On the other hand, the typical muon deflection angle is a function of the $Z^{\prime}$ mass, scaling as $\psi_{\mu^{\prime}} \simeq m_{Z^{\prime}} / E_{\mu}$ for a fixed incoming muon energy. Thus, the total cross section calculated for the deflected muon is sensitive to the maximum angle if $\psi_{\mu^{\prime}}^{\max } \gtrsim m_{Z^{\prime}} / E_{\mu}$. For this reason, in this case we integrate over the full parameter space of the muon and $Z^{\prime}$ without restricting the outgoing particles angles. In particular, for Fig. 7 the integration ranges are $m_{Z^{\prime}} / E_{\mu}<x<1-$ $m_{\mu} / E_{\mu}$ and $0<\theta_{Z^{\prime}}<\pi$ and $m_{\mu} / E_{\mu}<y<1-m_{Z^{\prime}} / E_{\mu}$ and $0<\psi_{\mu^{\prime}}<\pi$ for $Z^{\prime}$ and the muon, respectively. The relative error of both cross sections with respect to the exact cross section integrated over $x$ and $\theta$ for all the masses considered here is below $2 \%$ for energies above $10 \mathrm{GeV}$.
The total cross section derived integrating the muon fractional energy $y$ and the muon recoil angle $\psi_{\mu^{\prime}}$ is shown in Fig. 8 in IWW and WW approximations for both cases $\psi_{\mu^{\prime}}^{\max }=0.1$ and $\psi_{\mu^{\prime}}^{\max }=\pi$. The result is compared to the exact tree-level cross section integrated over $x$ and $\theta_{Z^{\prime}}$. Differences with respect to the exact values are negligible in the case of WW. However, in the low energy region, especially for higher masses, the differences become significant. In order not to restrict regions the scattered muon cross section has been integrated in a large recoil muon angle range $\left(\psi_{\mu^{\prime}}^{\max }=\pi\right.$ ) (see magenta and orange dotted lines in Fig. 8). The result is compatible with the one shown in Fig. 2.

\section{PROJECTED SENSITIVITIES TO THE MIXING STRENGTH}

The sensitivity of the experiment to $Z^{\prime}$ is calculated according to [10]. In particular, the expected number of $Z^{\prime}$, 

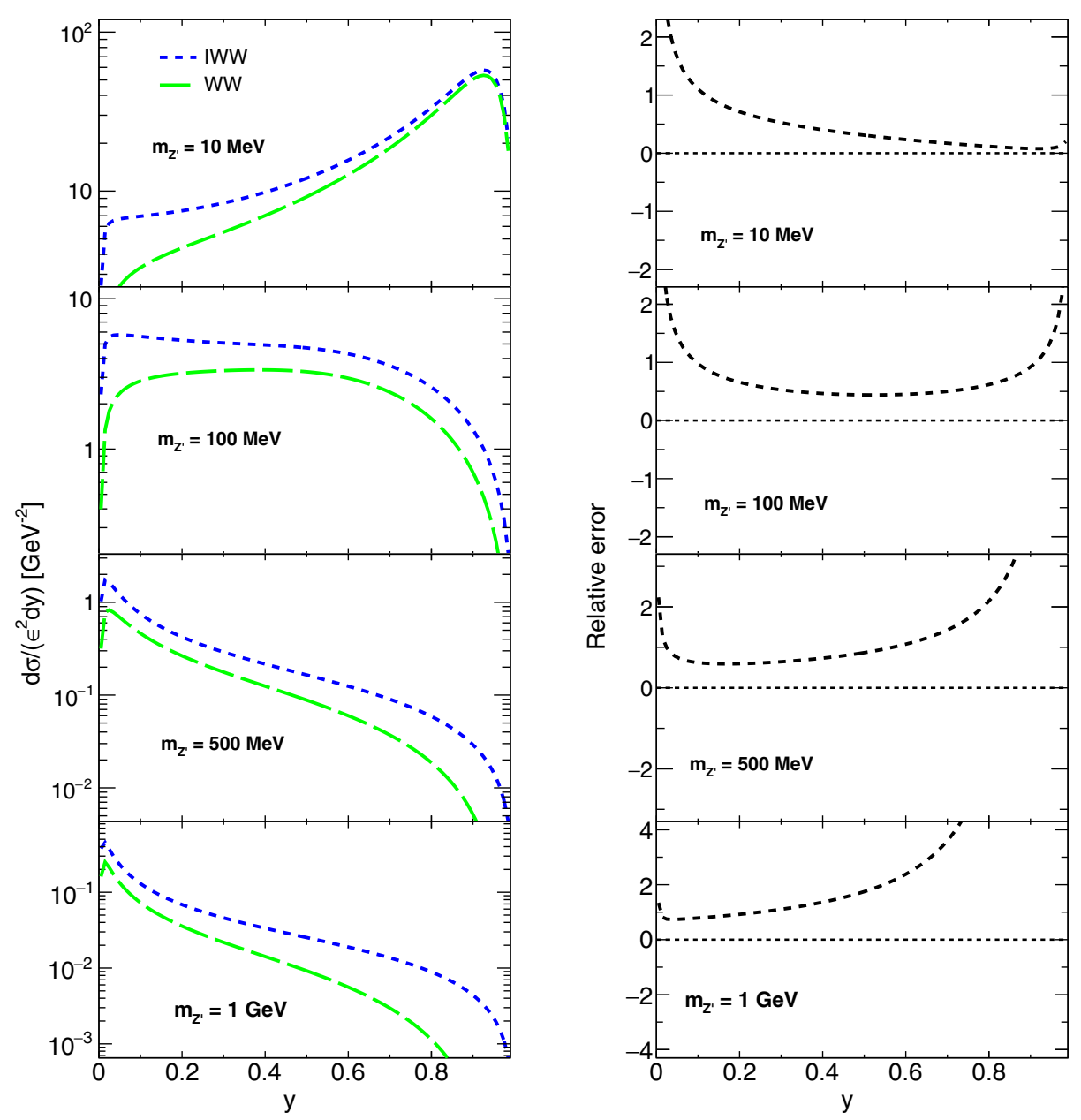

FIG. 6. Left: differential cross section as a function of $y$ for IWW (blue dashed line) and WW (green line) for different $Z^{\prime}$ masses. Right: relative error between IWW and WW defined as $\left(\mathcal{O}_{\text {IWW }}-\mathcal{O}_{\mathrm{WW}}\right) / \mathcal{O}_{\mathrm{WW}}$. The angle of muon deflection is integrated in the range $\psi \in[0,0.1]$.

$N_{Z^{\prime}}^{(\bar{\nu} \nu)}$ produced through muon bremsstrahlung and decaying invisibly to SM neutrinos, $Z^{\prime} \rightarrow \bar{\nu} \nu$, is given by

$$
\begin{aligned}
N_{Z^{\prime}}^{(\bar{\nu} \nu)}= & N_{\mathrm{MOT}} \cdot \frac{\rho \mathcal{N}_{A}}{A} \cdot \sum_{i} \Delta L_{i} \cdot \sigma_{\mathrm{tot}}^{Z^{\prime}}\left(E_{\mu}^{i}\right) \\
& \cdot \operatorname{Br}\left(Z^{\prime} \rightarrow \bar{\nu} \nu\right)
\end{aligned}
$$

where $A$ is the atomic weight, $\mathcal{N}_{A}$ is the Avogadros number, $N_{\text {MOт }}$ is the number of muons on target, $\rho$ is the target density, $E_{\mu}^{i}$ is the muon energy at the $i$ th step in the target, $\Delta L_{i}$ is the step length of the muon path, and $\sigma_{\text {tot }}^{Z^{\prime}}$ is the total cross section of the $Z^{\prime}$ production; $\operatorname{Br}\left(Z^{\prime} \rightarrow \bar{\nu} \nu\right)$ corresponds to the branching ratio of $Z^{\prime}$ decaying invisibly to SM neutrinos [4].

In the NA64 $\mu$ facility it is assumed to utilize two, upstream and downstream, magnetic spectrometers allowing for precise measurements of momenta for incident and recoiled muons, respectively [4]. The muon missing energy signal of the reaction $\mu N \rightarrow \mu N Z^{\prime}, Z^{\prime} \rightarrow \bar{\nu} \nu$ is defined by a scattered muon energy cut $E_{\mu^{\prime}} \lesssim 80 \mathrm{GeV}$.

The projected sensitivities are then calculated at 90\% C.L. for the number of signal events, i.e., it is required that $N_{Z^{\prime}}^{(\bar{\nu} \nu)}>$ 2.3 events, assuming zero background. Figure 9 shows the projected sensitivities for both IWW and WW approaches in the plane $\left(m_{Z^{\prime}}, \epsilon\right)$ for $10^{12} 160 \mathrm{GeV}$ muons on target (MOTs), together with the values of $\epsilon$ necessary for the explanation of the $(g-2)_{\mu}$ anomaly. Those are obtained separately through numerical integration of Eq. (44) with GSL [24] and with robust MC simulations using the DMG4 simulation package [13]. It can be seen that in the low mass region, $m_{Z^{\prime}} \lesssim 10 \mathrm{MeV}$, the relative error between IWW and WW does not exceed 10\%, whereas it reaches about $40 \%$ at masses $m_{Z^{\prime}} \sim \mathcal{O}(1 \mathrm{GeV})$. It is also worth noting that more conservative sensitivity lines are obtained with the realistic MC simulations, as a result of simulating the full muon physics within the active target, with about $\lesssim 10 \%$ relative error with respect to the numerically integrated results. 

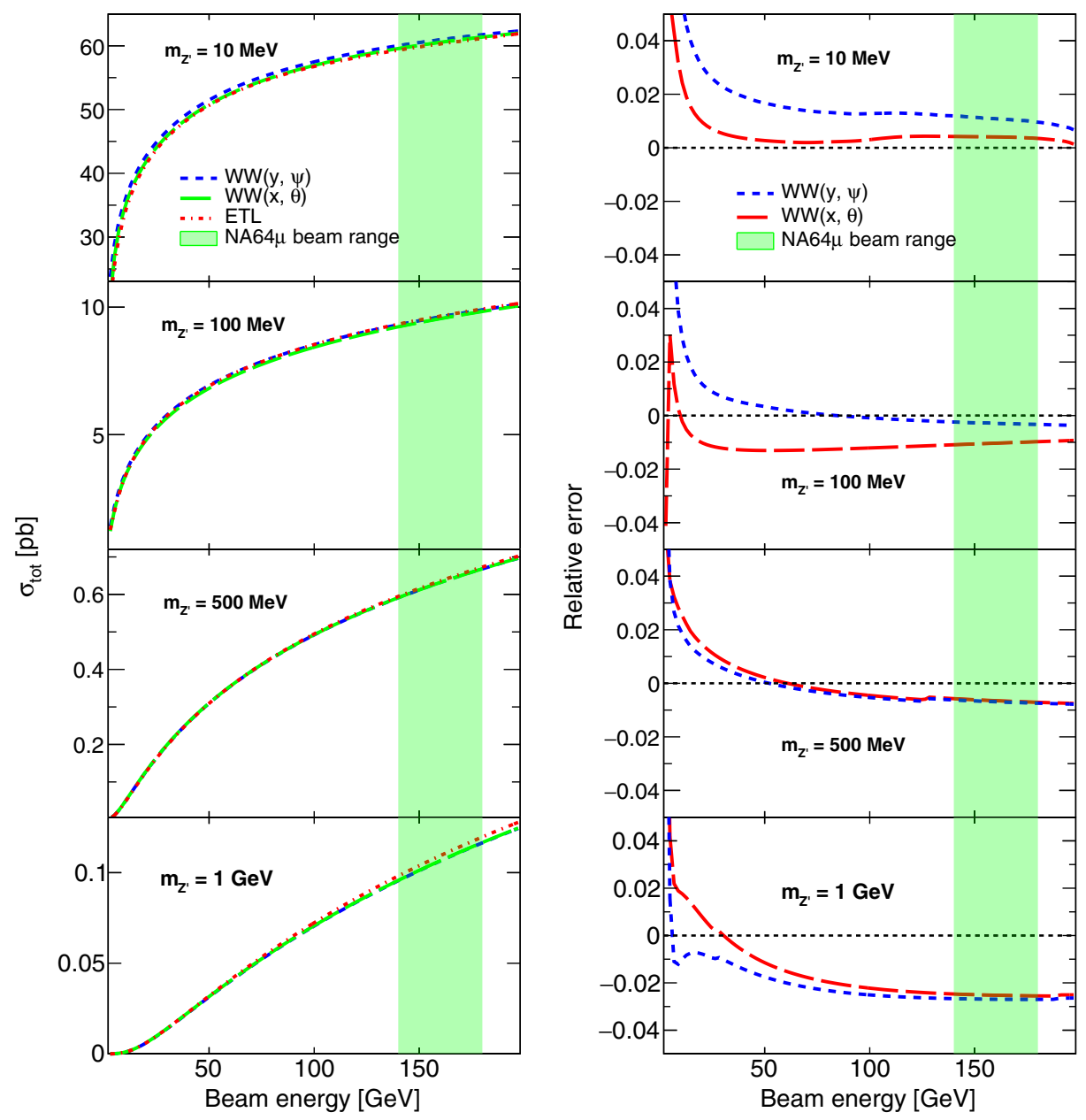

FIG. 7. Left: total cross section as a function of the beam energy for WW expressions integrated over $y, \psi$ (blue dashed line), $x, \theta$ (green line), and ETL integrated over $x, \theta$ (red dotted line). Right: relative error between WW approximations and ETL as a function of the beam energy expressed as $\left(\mathcal{O}_{\mathrm{WW}}-\mathcal{O}_{\text {exact }}\right) / \mathcal{O}_{\text {exact }}$. The typical NA64 $\mu$ beam energy range is shown in green. The angles are integrated in the ranges $\theta \in[0, \pi]$ and $\psi \in[0, \pi]$, the benchmark mixing strength is taken to be $\epsilon=10^{-4}$.

\section{SUMMARY}

In this work, we have derived, based on the work of [14], the differential and total cross sections for dark vector boson production in fixed target experiments through muon bremsstrahlung. We have shown that the commonly used improved Weizsäcker-Williams approximation differs significantly from the exact-tree-level calculations. On the other hand, the WW approach reproduces well the cross section at a level of $<5 \%$ in the high-energy beam regime, such as the one of NA64 $\mu$ experiment. However, for very low beam energies, where $E_{\mu} \sim m_{Z^{\prime}}$, the collinear regime is no longer valid and the phase-space approximations fail. We have also calculated the $Z$ double-differential cross section as a function of new variables, namely the scattered muon fractional energy and recoil angle, of particular importance for Monte Carlo simulations and estimates in missing momentum experiments. Although the ETL for those variables was not calculated, we have cross-checked our results against both WW approximation and ETL calculations as a function of the emitted vector boson variables and found that the newly derived WW cross section reproduces the total cross section with a good accuracy $(\lesssim 1 \%)$. Additionally, we developed an analytical expression of the photon flux in WW approximation to reduce computational time due to numerical integration. Finally, our calculations were used to derive possible projected sensitivities in missing momentum experiments with both numerical integration and a full realistic GEANT4based MC simulation. It was found that in the high mass region, IWW calculations differ from WW ones, and thus ETL, by as much as $40 \%$, overestimating the sensitivity of muon beam fixed target experiments. Our results demonstrate the potential of these experiments to explore a broad coupling and mass parameter space region of dark vector bosons such as $Z^{\prime}\left(A^{\prime}\right)$, including the very interesting muon $(g-2)$ unexplored region. 

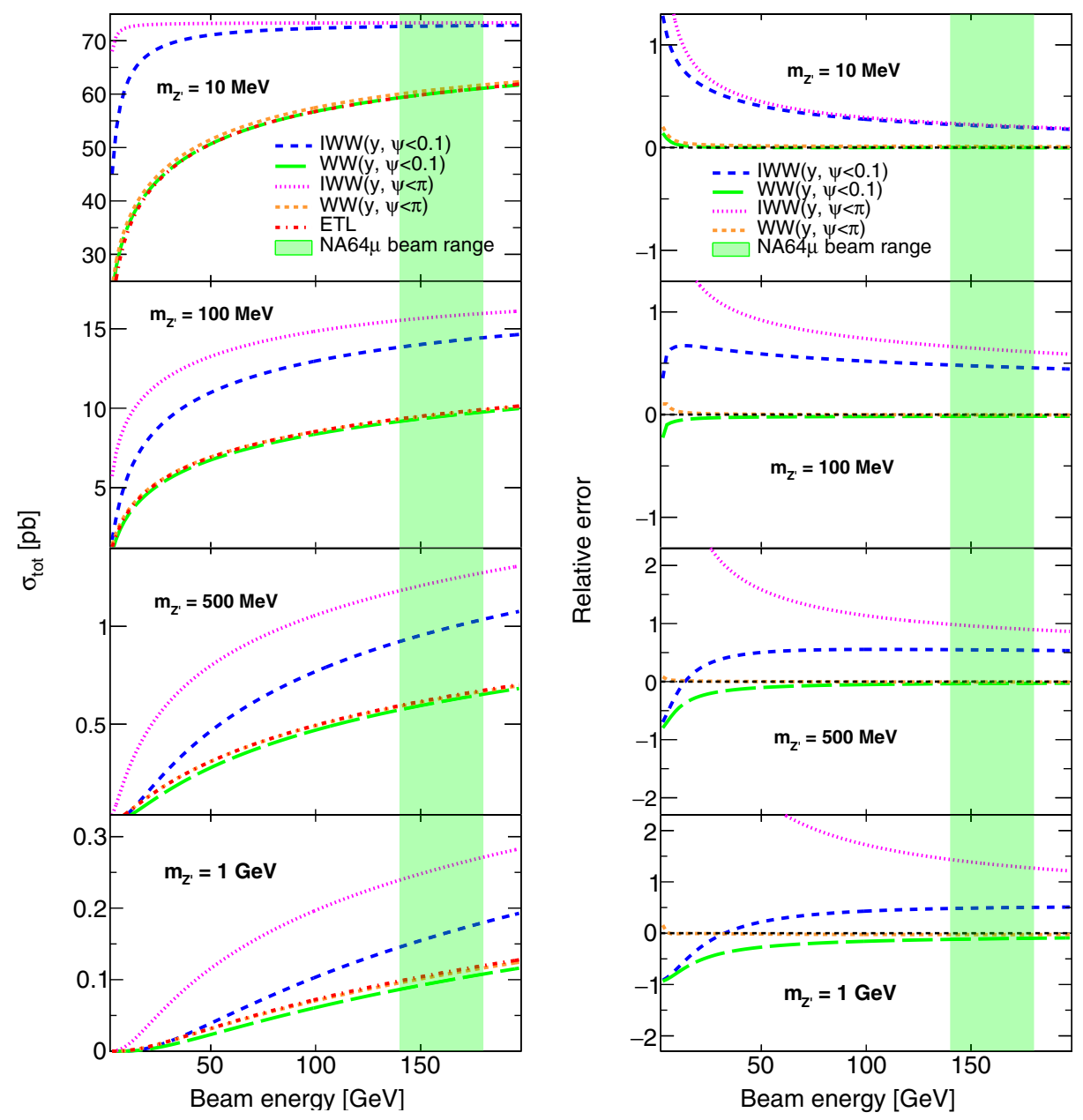

FIG. 8. Left: total cross section as a function of the beam energy for IWW (blue/magenta dashed line), WW (green/orange line) expressions, and ETL (red dotted line) integrated in the ranges $0<\psi_{\mu}<0.1$ (blue and green) and $0<\psi_{\mu}<\pi$ (magenta and orange). Right: relative error between WW, IWW approximations, and ETL as a function of the beam energy expressed as $\left(\mathcal{O}_{\mathrm{WW}}-\mathcal{O}_{\text {exact }}\right) / \mathcal{O}_{\text {exact }}$. The typical NA64 $\mu$ beam energy range is shown in green. The benchmark mixing strength is taken to be $\epsilon=10^{-4}$.
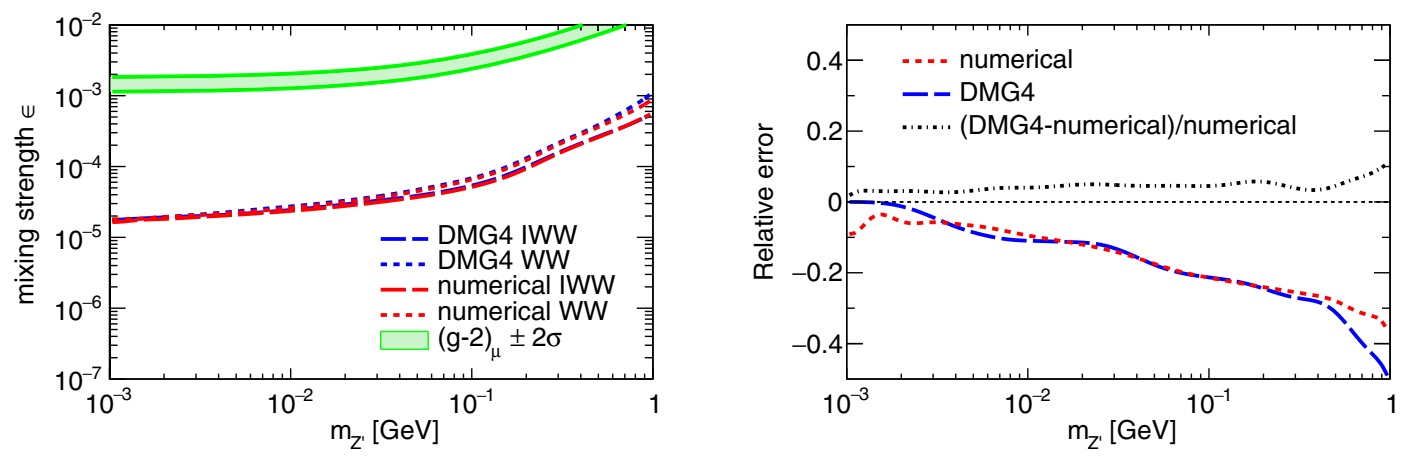

FIG. 9. Left: projected sensitivity in the $\left(m_{Z^{\prime}}, \epsilon=g^{\prime} / \sqrt{4 \pi \alpha}\right)$ phase space obtained through both MC simulations with DMG4 package [13] (blue lines) and GSL [24] numerical integration (red lines) for $Z^{\prime}$. The limits are calculated at $90 \%$ C.L. for $10^{12} \mathrm{MOT}$ and $160 \mathrm{GeV}$. Also shown is the $(g-2)_{\mu}$ favored band within $\pm 2 \sigma$. No other experimental constraints on the searches for $Z^{\prime}$ vector boson are included as those are beyond the scope of this paper. NA64 $\mu$ experiment is used only as a benchmark scenario to define the kinematic regime (for a detailed discussion, see [7,11]). Right: relative error between IWW and WW defined as $\left(\mathcal{O}_{\text {IWW }}-\mathcal{O}_{\mathrm{WW}}\right) / \mathcal{O}_{\mathrm{WW}}$. Is also shown the average relative error between MC simulations and numerical integration, defined as $\left(\mathcal{O}_{\text {DMG4 }}-\mathcal{O}_{\text {numerical }}\right) / \mathcal{O}_{\text {numerical }}$. 


\section{ACKNOWLEDGMENTS}

We acknowledge the members of the NA64 collaboration for fruitful discussions, in particular, S. N. Gninenko, N. V. Krasnikov, and E. Depero. The work of P. Crivelli, L. Molina Bueno, and H. Sieber is supported by ETH Zrich and SNSF Grants No. 186181, No. 186158, and No. 197346 (Switzerland). The work of D. V. Kirpichnikov on MC simulation of $Z^{\prime}$ emission is supported by the Russian Science Foundation RSF Grant No. 21-12-00379.

[1] B. Abi et al. (Muon g-2 Collaboration), Measurement of the Positive Muon Anomalous Magnetic Moment to 0.46 ppm, Phys. Rev. Lett. 126, 141801 (2021).

[2] T. Aoyama et al., The anomalous magnetic moment of the muon in the Standard Model, Phys. Rep. 887, 1 (2020).

[3] G. Lanfranchi, M. Pospelov, and P. Schuster, The Search for Feebly-Interacting Particles, arXiv:2011.02157.

[4] S. N. Gninenko, N. V. Krasnikov, and V. A. Matveev, Muon $g-2$ and searches for a new leptophobic sub-GeV dark boson in a missing-energy experiment at CERN, Phys. Rev. D 91, 095015 (2015).

[5] S. N. Gninenko and N. V. Krasnikov, The muon anomalous magnetic moment and a new light gauge boson, Phys. Lett. B 513, 119 (2001).

[6] C.-Y. Chen, M. Pospelov, and Y.-M. Zhong, Muon beam experiments to probe the dark sector, Phys. Rev. D 95, 115005 (2017).

[7] S. N. Gninenko and N. V. Krasnikov, Probing the muon $(g-2)_{\mu}$ anomaly, $L_{\mu}-L_{\tau}$ gauge boson and Dark Matter in dark photon experiments, Phys. Lett. B 783, 24 (2018).

[8] D. V. Kirpichnikov, V. E. Lyubovitskij, and A. S. Zhevlakov, Implication of hidden sub-GeV bosons for the $(g-2)_{\mu}$, ${ }^{8} \mathrm{Be}-{ }^{4} \mathrm{He}$ anomaly, proton charge radius, EDM of fermions, and dark axion portal, Phys. Rev. D 102, 095024 (2020).

[9] D. W. P. Amaral, D. G. Cerdeño, A. Cheek, and P. Foldenauer, Distinguishing $U(1)_{L_{\mu}-L_{\tau}}$ from $U(1)_{L_{\mu}}$ as a solution for $(g-2)_{\mu}$ with neutrinos, arXiv:2104.03297.

[10] D. Banerjee et al. (NA64 Collaboration), Proposal for an experiment to search for dark sector particles weakly coupled to muon at the SPS, Report No. CERNSPSC:2019-002/SPSC-P-359 (2019).

[11] Y. Kahn, G. Krnjaic, N. Tran, and A. Whitbeck, $M^{3}$ : A new muon missing momentum experiment to probe $(g-2)_{\mu}$ and dark matter at Fermilab, J. High Energy Phys. 09 (2018) 153.

[12] S. Agostinelli et al. (GEANT4 Collaboration), Geant4-a simulation toolkit, Nucl. Instrum. Methods Phys. Res., Sect. A 506, 250 (2003).

[13] M. Bondi, A. Celentano, R. R. Dusaev, D. V. Kirpichnikov, M. M. Kirsanov, N. V. Krasnikov, L. Marsicano, and D. Shchukin, Fully Geant 4 compatible package for the simulation of Dark Matter in fixed target experiments, Comput. Phys. Commun. 269, 108129 (2021).
[14] Y.-S. Liu and G. A. Miller, Validity of the WeizsäckerWilliams approximation and the analysis of beam dump experiments: Production of an axion, a dark photon, or a new axialvector boson, Phys. Rev. D 96, 016004 (2017).

[15] S. N. Gninenko, D. V. Kirpichnikov, M. M. Kirsanov, and N. V. Krasnikov, The exact tree-level calculation of the dark photon production in highenergy electron scattering at the CERN SPS, Phys. Lett. B 782, 406 (2018).

[16] Y.-S. Liu, D. McKeen, and G. A. Miller, Validity of the Weizsäcker-Williams approximation and the analysis of beam dump experiments: Production of a new scalar boson, Phys. Rev. D 95, 036010 (2017).

[17] T. Beranek and M. Vanderhaeghen, Study of the discovery potential for hidden photon emission at future electron scattering fixed-target experiments, Phys. Rev. D 89, 055006 (2014).

[18] T. Beranek, H. Merkel, and M. Vanderhaeghen, Theoretical framework to analyze searches for hidden light gauge bosons in electron scattering fixed target experiments, Phys. Rev. D 88, 015032 (2013).

[19] V. Shtabovenko, R. Mertig, and F. Orellana, FeynCalc 9.3: New features and improvements, Comput. Phys. Commun. 256, 107478 (2020).

[20] V. Shtabovenko, R. Mertig, and F. Orellana, New developments in FeynCalc 9.0, Comput. Phys. Commun. 207, 432 (2016).

[21] W. R. Inc., Mathematica, Version 12.2, champaign, IL, 2020.

[22] K. J. Kim and Y.-S. Tsai, Improved Weizsäcker-Williams Method and Its Application to Lepton and W-Boson Pair Production, Phys. Rev. D 8, 3109 (1973).

[23] J. D. Bjorken, R. Essig, P. Schuster, and N. Toro, New fixedtarget experiments to search for dark gauge forces, Phys. Rev. D 80, 075018 (2009).

[24] M. Galassi et al., GNU Scientific Library Reference Manual -Third Edition (Network Theory Ltd., Surrey, 2009).

[25] D. E. Groom, N. V. Mokhov, and S. I. Striganov, Muon stopping power and range tables $10 \mathrm{MeV}-100 \mathrm{TeV}$, Atom. Data Nucl. Data Tabl. 78, 183 (2001).

[26] S. Kelner et al., About Cross Section for High-Energy Muon Bremsstrahlung, About Cross Section for High-Energy Muon Bremsstrahlung, Preprint MEPhI 024-95 (MEphI, Moscow, 1995). 